\title{
Protective effects of Akkermansia muciniphila on cognitive deficits and amyloid pathology in a mouse model of Alzheimer's disease
}

\author{
Zihao Ou', Lulu Deng ${ }^{1}$, Zhi Lu', Feifan Wu ${ }^{1,2}$, Wanting Liu', Dongquan Huang ${ }^{1}$ and Yongzheng Peng (1) ${ }^{1,3}$
}

\begin{abstract}
Objective: Alzheimer's disease (AD) is a global health problem without effective methods to alleviate the disease progression. Amyloid $\beta$-protein $(A \beta)$ is widely accepted as a key biomarker for AD. Metabolic syndromes, including obesity and insulin resistance, are key high risk factors for AD. Akkermansia muciniphila (Akk), the only representative human gut microbe in the genus Verrucomicrobia, can prevent the weight gain caused by a high-fat diet, repair the damaged integrity of the intestinal epithelium barrier, reduce endotoxin levels in blood and improve insulin resistance. The aim of this study is to explore the impact of Akk administration in AD model mice in different diets.
\end{abstract}

Methods: APP/PS1 mice were fed either a normal chow diet or a high-fat diet and were treated with Akk by gavage each day for 6 months. The impacts of Akk on glucose metabolism, intestinal barrier and lipid metabolism in the mouse model of AD were determined. Changes in brain pathology and neuroethology were also analyzed.

Results: Akk effectively reduced the fasting blood glucose and serum diamine oxidase levels, and alleviated the reduction of colonic mucus cells in APP/PS1 mice. After treatment with Akk, the APP/PS1 mice showed obviously reduced blood lipid levels, improved hepatic steatosis and scapular brown fat whitening. Moreover, Akk promoted the reduction of A $\beta$ 40-42 levels in the cerebral cortex of APP/PS1 mice, shortened the study time and improved the completion rate in $Y$-maze tests.

Conclusion: Akk effectively improved glucose tolerance, intestine barrier dysfunction and dyslipidemia in AD model mice. Our study results suggested that Akk could delay the pathological changes in the brain and relieve impairment of spatial learning and memory in AD model mice, which provides a new strategy for prevention and treatment of AD.

\section{Introduction}

Alzheimer's disease $(\mathrm{AD})$ is a disease with generalized dementia, such as memory impairment, loss of visual skills, performance impairment and behavioral changes. $\mathrm{AD}$ is characterized by serious cognitive impairment and significant pathological changes in the brain. Amyloid

Correspondence: Yongzheng Peng (yzpeng1981@126.com)

'Department of Laboratory Medicine, Zhu Jiang Hospital, Southern Medical University, 510282 Guangzhou, Guangdong, China

${ }^{2}$ Jiangmen Central Hospital, Affiliated Jiangmen Hospital of Sun Yat-sen University, 529000 Jangmen, Guangdong, China

Full list of author information is available at the end of the article $\beta$-protein $(A \beta)$ is a peptide produced by $A \beta$ precursor protein (APP) through proteolysis of $\beta$ - and $\gamma$-secretase. $A \beta 40$ and $A \beta 42$ have strong toxicity, and they are easier to aggregate and cause neurotoxicity effects ${ }^{1}$, which is widely accepted as a key biomarker for AD. Current treatments for AD relieve only the symptoms, but cannot alleviate disease progression ${ }^{2}$. As such, early diagnosis is the most effective treatment method ${ }^{3}$. In 2010, Alzheimer's Disease International (ADI) reported that costs of treatment and care for dementia worldwide had reached more than 818 billion dollars. Experts speculate that the population of AD patients could reach 30 million by $2050^{4}, 71 \%$ of 
which would be concentrated in developing countries, including China, India, South Asia and the western Pacific regions.

Recently, a large number of reports have indicated that metabolic syndromes, including obesity and insulin resistance, are key high risk factors for cognitive impairment and dementia ${ }^{5}$. The data suggested that young people who suffered from obesity or diabetes had a greater risk of dementia ${ }^{6-9}$. Type 2 diabetes also greatly increased the risk of $\mathrm{AD}$, and $\mathrm{A} \beta$ protein deposition competes with insulin for insulin receptors, causing insulin resistance ${ }^{10-13}$. In addition, animal experiments showed that metabolic syndrome, chronic low-grade inflammation in particular, could increase specific pathologic changes in $\mathrm{AD}^{13-15}$, and experts think that $\mathrm{AD}$ is a metabolic disease ${ }^{16-18}$. The pathogenesis of $\mathrm{AD}$ has not yet been clearly defined. Many studies have shown that the development of insulin resistance in the brain appears in $A D$ patients, which may be caused by $A \beta$ protein deposition, as it is associated with multiple pathological features ${ }^{19,20}$. Therefore, many scholars believed that a dysfunctional cerebral insulin pathway might be a partial cause of $\mathrm{AD}^{21}$.

Akkermansia muciniphila (Akk) is the only representative Verrucomicrobia of the human intestine microbes that can be cultured, and it can be detected easily by metagenomic analysis of the human intestine. Given that its abundance is closely related to human health, A. muciniphila has received increasing attention ${ }^{22}$. There is evidence that the abundance of $A$. muciniphila was significantly decreased in the intestines of patients with type 2 diabetes ${ }^{23}$ and $\mathrm{AD}$ model mice ${ }^{24}$. It has also been shown that $A$. muciniphila cannot only prevent weight gain in high-fat diet mice, but can also repair the damaged epithelial barrier integrity and improve endotoxemia $^{25,26}$. Therefore, the specific aim of this study was to evaluate whether Akk treatment could improve the health of animal models with $\mathrm{AD}$, in order to provide a new strategy for prevention and treatment of AD.

\section{Methods}

\section{Animal model}

APPswe/PS1dE9 (APP/PS1) double-transgenic mice and the parasite-free wild-type mice were purchased from the Model Animal Research Center of Nanjing University $^{27}$. In order to clarify whether Akk works only under the conditions of high-fat diet, 3-month-old male APP/PS1 mice were fed ad libitum either a normal chow diet or a high-fat diet (Catalog no. D12492, Research Diet, GDMLAC) for 6 months. Moreover, in order to verify that Akk has no harmful effect in normal mice, the WT mice were divided into two groups to receive Akk administration. Taking into account, the statistical analysis and the sample size required for subsequent experiments in this study, APP/PS1 mices were randomly assigned into 4 groups ( $n=10$ per group) and WT mices were randomly assigned into two groups ( $n=6$ per group). Mice were overnight fasted and anesthetized with pentobarbital sodium. Tissues (scapular fat, cerebral cortex, hippocampus, liver, colon) were isolated and preserved after blood collection. All animal experiments were approved by the Committee on the Use of Live Animals in Teaching and Research at the University of the Southern Medical University. This study selects the sample size based on the experimental quantity requirement and the animal experiment 3R principle. All animals are grouped according to the principle of randomization and all animal experiments were performed with blinding.

\section{Culture and administration of $A$. muciniphila}

The Chinese strain A. muciniphila GP01 was isolated from human stool, as previously described ${ }^{28}$, and cultured anaerobically in a basal mucin-based medium. The concentration of bacteria was calculated by measuring the OD values at $600 \mathrm{~nm}$. The experimental APP/PS1 mice were gavaged daily for 6 months with either $5 \times 10^{9} \mathrm{cfu}$ of A. muciniphila in $200 \mu \mathrm{L}$ sterile PBS or $200 \mu \mathrm{L}$ sterile PBS.

\section{MRI}

Mice were anesthetized with $1-3 \%$ isoflurane in $100 \%$ $\mathrm{O}_{2}$. Anesthetized mice were scanned in a 7T small animal MRI (PharmaScan70/16 US; BRUKER Biospin MRI $\mathrm{GmbH}$ ) fitted with sensitive surface coils and amplifier. Animal welfare was provided by employing a hot water circulation system and physiological monitoring. The imaging protocol included a T2 sequence with the following parameters: TR/TE:2500/35 ms; FoV: $15 \times 30 \mathrm{~mm}$; image size: $256 \times 256 \mathrm{~mm}$; Slice thickness: $0.7 \times 3.4 \mathrm{~mm}$; scan time: $2 \mathrm{~min} 4 \mathrm{~s}$. The size of the cerebral hemispheres and lateral ventricles on both sides of the mouse can be evaluated by MRI.

\section{Immunohistochemistry and histology}

Immunohistochemistry (IHC) was used to detect $A \beta$ protein deposition. The paraffin sections were deparaffinized $(4 \mu \mathrm{m})$ and dehydrated with an ethanol gradient, and antigen retrieval was accomplished by boiling in citric acid buffer for $15 \mathrm{~min}$. After washing with $1 \times \mathrm{PBS}(\mathrm{pH}$ 7.4), sections were incubated with $3 \% \mathrm{H}_{2} \mathrm{O}_{2}$ to quench endogenous peroxidase activity. Sections were rinsed, incubated with $3 \%$ normal goat serum in TBS for $30 \mathrm{~min}$, and incubated overnight at $4{ }^{\circ} \mathrm{C}$ with the primary antibody ( $\beta$-Amyloid D12B2, Abcam, Cambridge, MA, USA). Horseradish peroxidase-conjugated secondary antibody was used to bind the primary antibody and the complex was visualized using a stable diaminobenzidine (DABI) solution. The stained sections were dehydrated with graded alcohols and cleared in xylene. Periodic acid-Schiff 
(PAS) staining was used to detect colonic goblet cells. The colon samples were dehydrated with an ethanol gradient $(75,85,95,100 \%)$ and embedded in paraffin. The sections were cut $(4 \mu \mathrm{m})$ and processed with periodic acid (1\%) for 5 min, washed with distilled water, treated with Schiff's reagent for $5 \mathrm{~min}$, and washed again. The sections were stained with Harris haematoxylin for $2 \mathrm{~min}$. Hematoxylin-eosin (H\&E) staining was utilized to observe hepatic steatosis. The samples were fixed in $4 \%$ paraformaldehyde and embedded in paraffin. The sections were stained with $\mathrm{H} \& \mathrm{E}$ as routinely described. For quantification, five sections with the same reference position were chosen from each mouse for analysis using IMAGE-PRO PLUS 6.0 imaging software.

\section{Biochemical assays and ELISA}

Serum levels of total cholesterol (Chol), triglycerides (TG), alanine aminotransferase (ALT) and aspartate transaminase (AST) were determined using a Roche Modular P 800 Analyzer. Serum levels of diamine oxidase (DAO) (CUSABIO) and levels of A $\beta 40$ and A 342 (Invitrogen) in the cerebral cortex were measured with ELISA kits.

\section{Glucose tolerance test}

APP/PS1 mice were fasted for $12 \mathrm{~h}$, and fasting blood glucose was measured. Glucose $(2 \mathrm{~g} / \mathrm{kg})$ was then delivered orally and blood glucose levels were determined with a glucometer (Abbott Laboratories, Abbott Park, IL, USA) at $15,30,60,90$ and $120 \mathrm{~min}$.

\section{Open-field and Y-maze tests}

The open-field test arena was constructed of a plastic chamber $(30 \times 50 \times 25 \mathrm{~cm})$. The camera, connected to a computer, was placed above the chamber to monitor the distance traveled and the time devoted to the center and peripheral areas of the arena. The mice were placed in the center of the arena and allowed to explore for $10 \mathrm{~min}$. Before each test, the box was cleaned with $75 \%$ alcohol. The Y-maze was done on a black-plastic board and each arm was $15 \mathrm{~cm}$ long, $14 \mathrm{~cm}$ high and $5 \mathrm{~cm}$ wide, and positioned at equal angles. Mice were located at the end of one arm and allowed to move freely through the maze during a 2-min session. Arm entry was considered complete when the hind paws of the mouse were completely placed in the arm. Each mouse trained 20 times a day, training for up to 3 days, until the mouse completed the test nine of ten times correctly. After $24 \mathrm{~h}$, each mouse was tested ten times. The whole process was recorded, which included the total time and the correct and incorrect times. Achieving the standard: 9 or more correct responses (correct response rate $290 \%$ ) for 10 consecutive times are set as the learning standards. Observation indicators: 1 . The total time required for training, which reflects the learning ability of mice; 2 . Memory retention (reproduction) test: After $24 \mathrm{~h}$ of learning test, test the same method 10 times. The accuracy reflects the memory ability of mice.

\section{Western blot analyses}

Mouse hippocampi were homogenized in chilled TBS supplemented with a protease- and phosphatase inhibitor cocktail (Thermo Fisher Scientific, Waltham, MA, USA). Protein concentrations of the homogenates were determined by BCA assay (CWBIO). Samples were electrophoretically separated on 10 or $12 \%$ SDS-PAGE gels and transferred to PVDF membranes (Millipore, Bedford, MA, USA). Membranes were blocked with $5 \%$ nonfat milk in TBS containing $0.1 \%$ Tween-20 (TBS-T) for $1 \mathrm{~h}$ and probed with primary antibodies (Anti-UCP-1 antibody ab10983) at $4{ }^{\circ} \mathrm{C}$ overnight. The membranes were subsequently developed with the corresponding horseradish peroxidase-conjugated secondary antibody and ECL kit (Thermo Fisher Scientific). Densitometry quantification of the protein bands was analyzed using IMAGE-PRO PLUS 6.0 imaging software.

The mixture of proteins was extracted from scapular brown adipose tissues in RIPA buffer (0.5\% NP40, 0.1\% sodium doxycycline, $150 \mathrm{mM} \mathrm{NaCl}, 50 \mathrm{mM}$ Tris. $\mathrm{HCl}[\mathrm{pH}$ 7.4]) containing a complete protease inhibitor cocktail (Abcam). PVDF membranes were probed simultaneously with primary antibodies of UCP1 (Abcam) and $\beta$-tubulin (Abcam). The protein bands were visualized with ECL chemiluminescence reagents (Thermo Fisher Scientific) and quantified using IMAGE-PRO PLUS 6.0 imaging software.

\section{Real-time PCR}

For analysis of Akk abundance in feces, fecal bacterial DNA was extracted by Magen Hipure Stool DNA kit. After adjusting the DNA concentration, real-time PCR was conducted using the PrimeScript ${ }^{\mathrm{TM}}$ RT reagent Kit (Cat No. RR820A, Takara, Dalian) following the manufacturer's protocol in a LightCycle480 real-time PCR System (Roche, Germany). The procedure included predenaturation of DNA samples at $95^{\circ} \mathrm{C}$ for $30 \mathrm{~s}$, then 40 cycles of denature at $95^{\circ} \mathrm{C}$ for $5 \mathrm{~s}$ and anneal at $60^{\circ} \mathrm{C}$ for $30 \mathrm{~s}$, melted at $95^{\circ} \mathrm{C}$ for $5 \mathrm{~s}$ and $60^{\circ} \mathrm{C}$ for $1 \mathrm{~min}$, finally cooled at $50{ }^{\circ} \mathrm{C}$ for $30 \mathrm{~s}$. The fluorescent product was detected in the last step of each cycle. Relative quantification was calculated with $2^{-\Delta \Delta \mathrm{Ct}}$, the results were normalized to $16 \mathrm{~S}$ rRNA gene.

The primers were: Akkermansia muciniphila gene, Forward 5'-AGCCTGAACGCATTATCCGC-3'; 
Reverse 5'-AGGGCAGTTCTTCCAGCGTA-3'. 16S rRNA gene,

Forward 5'-GTGYCAGCMGCCGCGGTAA-3'; Reverse 5'-GGACTACNVGGGTWTCTAAT-3'.

\section{Statistical analysis}

All samples were involved in statistical analysis. All analyses were performed with the Statistical Package for Social Sciences version 19.0 (SPSS) (IBM Corp, Armonk, NY, USA). Data were expressed as mean \pm SEM. Statistical significance was determined by Student's $t$-test (for comparison of two experimental groups) or one-way ANOVA (for comparison of three or more experimental groups). Comparisons with $p<0.05$ were considered statistically significant.

\section{Results}

Akk effectively regulated the glucose homeostasis and damage to the intestinal barrier in APP/PS1 mice

To assess the effects of Akk on the intestinal barrier and glucose homeostasis in an AD model mouse, we compared in the Akk-treated and PBS-treated APP/ PS1 mice fed either a normal chow diet (NCD) or a high-fat diet (HFD). In APP/PS1 mice treated with PBS, we found that the fasting blood glucose level was higher, and OGTT results also showed an increase in area of the AUC (Fig. $1 \mathrm{~d}-\mathrm{f}, p<0.05$, respectively). In striking contrast, significantly reduced levels of fasting blood glucose in APP/PS1 mice appeared after Akk treatment, and impaired glucose tolerance was improved, but only the high-fat diet group showed statistical differences (Fig. $1 \mathrm{~d}-\mathrm{f}, p<0.05$, respectively). These results demonstrated that in APP/PS1 mice, Akk reduced fasting blood glucose levels and regulated glucose metabolism disorders.

Next, we explored the possible role of Akk in intestinal barrier function in APP/PS1 mice. Diamine oxidase (DAO) is a highly active intracellular enzyme in the intestinal upper villus in human and mammals, has a role in the metabolism of histamine and various polyamines, and is closely related to nucleic acid and protein synthesis in mucosal cells (Fig. 1a, b, $p<0.05$ ). DAO can reflect the integrity of the intestinal mechanical barrier and the degree of damage. Our results showed that the colonic mucus cells in PBS-treated APP/PS1 mouse were greatly reduced, and serum DAO levels were significantly increased, as demonstrated by PAS staining and ELISA (Fig. 1c, $p<0.05$ ). The results demonstrated that intestinal barrier function in APP/PS1 mice was disorder. However, after treatment with Akk, there was no significant decrease in colonic mucus cells, and serum DAO levels were significantly lower than those in the PBS group. Only the NCD groups showed statistical difference, indicating that Akk could repair the intestinal barrier dysfunction in APP/PS1 mice.

\section{Akk effectively relieved hyperlipidemia and whitening of brown adipose tissue in APP/PS1 mice}

We explored the effects of Akk on lipid metabolism in AD model mice. In APP/PS1 mice, there was no obvious change in food intake or body weight gain between the Akk and PBS treatment groups (Fig. 2a, b). In addition, 9month-old APP/PS1 mice developed significant lipid metabolism disorders, evidenced by increasing levels of serum Chol and TG (Fig. 2c, d, $p<0.05$, respectively), and fat accumulation in the liver, which was similar between the high-fat diet group and normal diet group. Interestingly, lipid metabolism disorders in APP/PS1 mice treated with Akk were significantly improved, showing a decrease in serum Chol and TG levels, and hepatic fat scores showed ameliorative hepatic steatosis (Fig. 2e, f, $p<0.05$, respectively). It is worth noting that there were no differences in serum ALT and AST levels between six groups (Supplementary Fig. 2). Brown adipose tissue (BAT) possessed the anti-obesity potential, which was characterized by large numbers of mitochondria, where uncoupling protein 1 (UCP1) possesses the ability to utilize free fatty acids and glucose for heat generation (non-shivering thermogenesis). Because the cells are rich in tiny lipid droplets, brown adipocytes take on a multilocular appearance after H\&E staining. We found that the scapular brown adipocytes in APP/PS1 mice changed from multilocular adipocytes to unilocular adipocytes, shown as whitening of the brown fat. After Akk treatment, the whitening phenomenon of brown fat was obviously weakened, and the number of unilocular adipocytes in scapular brown fat tissue was also reduced (Fig. 2g). Moreover, after Akk treatment, the expression of UCP1 in the brown fat of APP/PS1 mice was increased, suggesting a more active themogenesis. High-fat diet aggravated the whitening of brown adipocytes in APP/PS1 mice, but did not reduce the thermogenic activity in brown fat (Fig. $2 \mathrm{~h}, \mathrm{j}, p<0.05$, respectively). These results indicated that Akk improved hyperlipidemia and hepatic steatosis in APP/PS1 mice, alleviated the whitening of brown fat and maintained thermogenic activity. In summary, Akk supplementation can regulate lipid metabolism disorders in APP/PS1 mice.

\section{Akk reduced $A \beta$ plaque deposits and $A \beta$ levels in brains of APP/PS1 mice}

Analysis of $A \beta$ protein deposition by immunohistochemistry showed that APP/PS1 mice had more A $\beta$ plaques in the hippocampus (Fig. 3a).The area of $A \beta$ protein deposition increased in APP/PS1-HFD-PBS mice, but was reduced when treated with Akk, although there was no statistically significant difference (Fig. 3b) In 


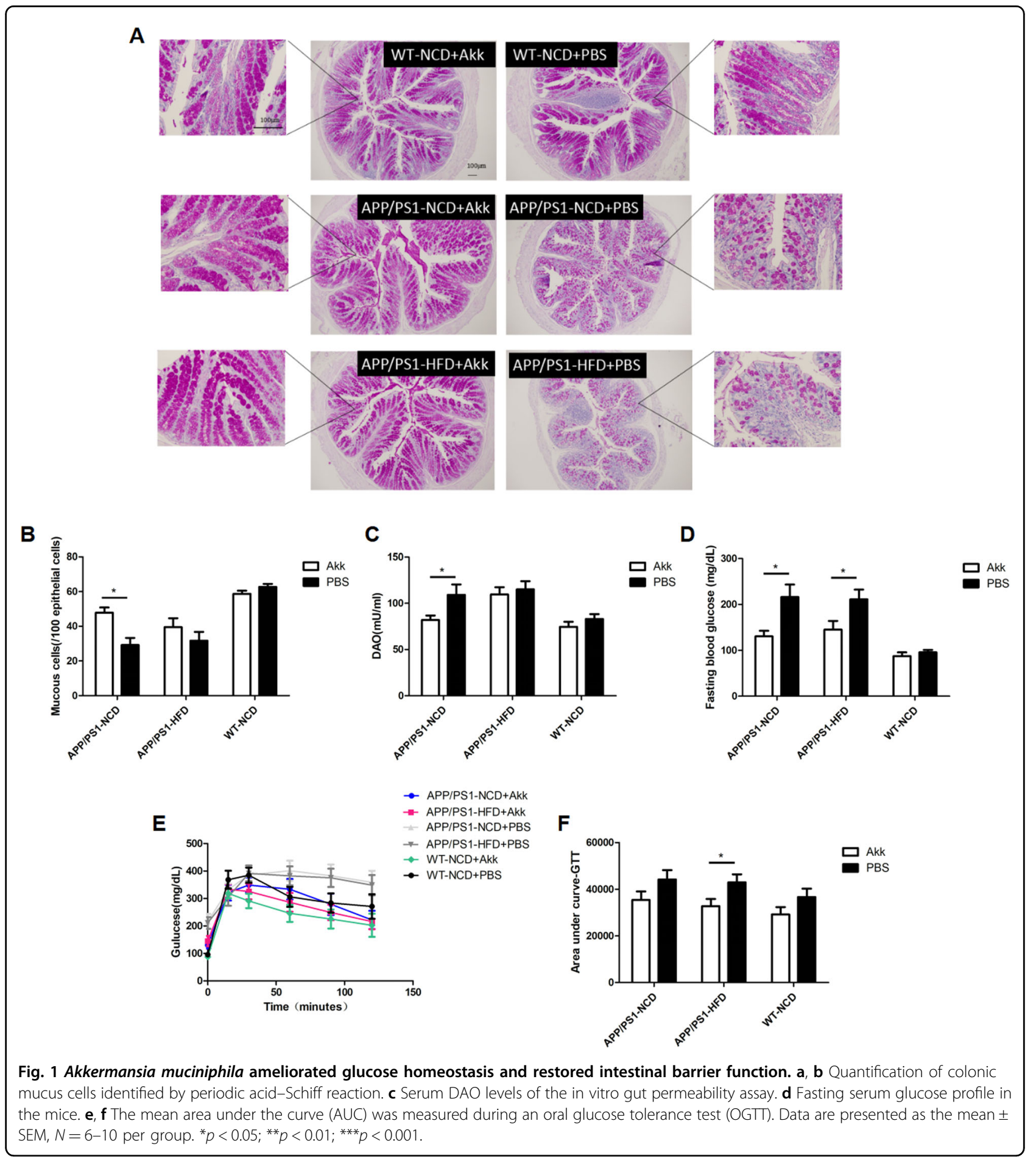

addition, we also analyzed soluble $A \beta 40$ and $A \beta 42$ proteins via ELISA. In APP/PS1-Akk mice, we detected a significant reduction in soluble $A \beta 40$ and $A \beta 42$ levels compared with APP/PS1-PBS mice (Fig. 3c, d, $p<0.01$; $p<0.05$, respectively). MRI showed that there was not significant atrophy in the mouse brains (Supplementary
Fig. 3), indicating that differences between the APP/PS1Akk mice and the APP/PS1-PBS mice were not caused by changes in brain structure. The results suggested that Akk intervention could reduce $A \beta$ plaque deposits and $A \beta$ levels in the brains of APP/PS1 mice independent of changes in brain structure. 


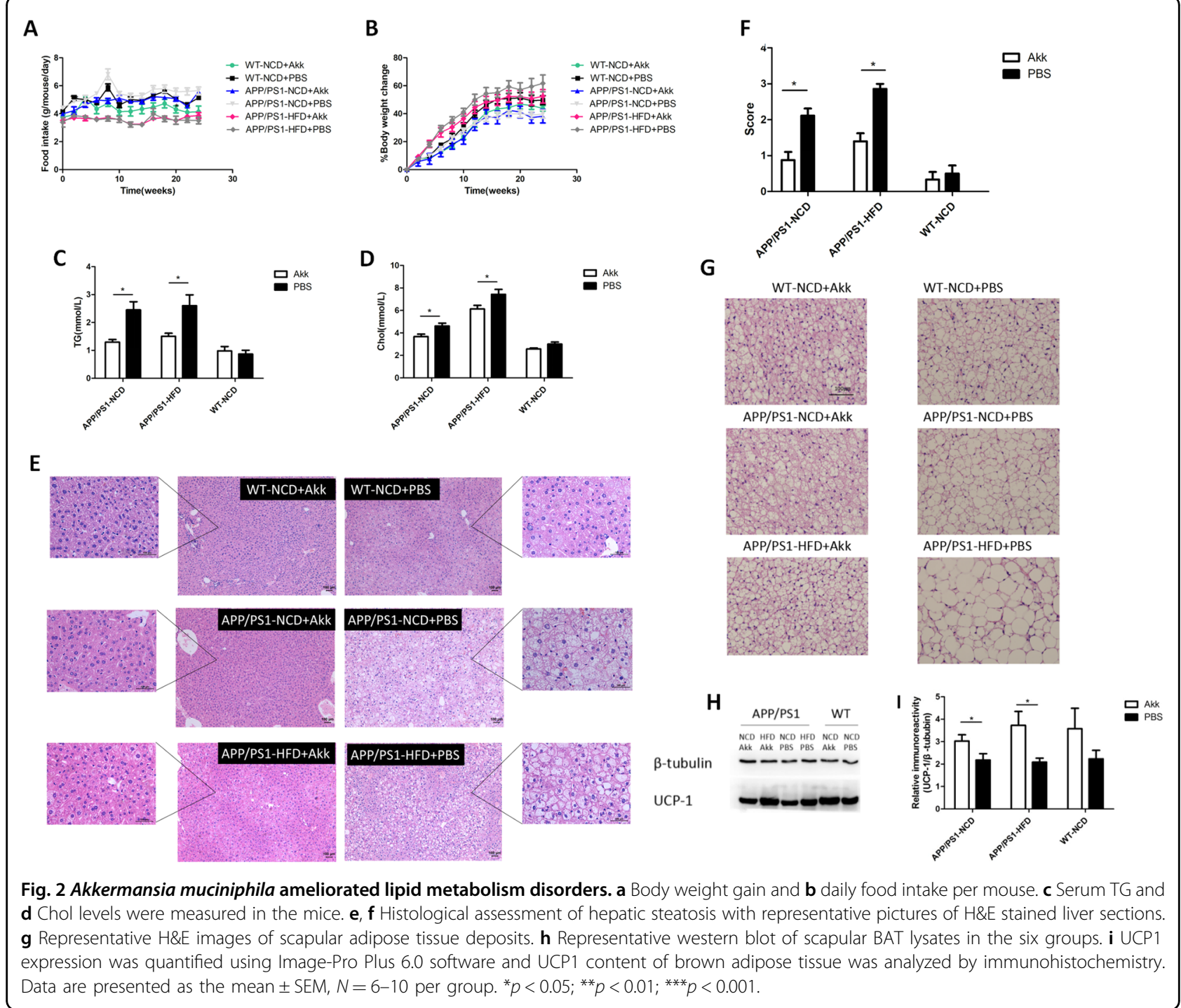

\section{Akk improved impaired cognition and anxiety-related behaviors in APP/PS1 mice}

In order to investigate the learning and memory capabilities of APP/PS1 mice, the Y-maze test was performed. Before the intervention, all mice received the Y-maze test for a maximum of 3 days. However, the APP/PS1-PBS mice spent more time than WT-PBS mice when training. After 6 months of Akk treatment, APP/PS1 mice required significantly shorter learning time than PBS-treated group (Fig. 4a, $p<0.001$ ). These data indicated that Akk could alleviate the damage to learning ability in APP/PS1 mice. After $24 \mathrm{~h}$ of practice in the Y-maze, each mouse was controlled for 10 times. The APP/PS1-PBS mice had rates of correct maze solving that were significantly lower than those in the other groups (Fig. $4 b, p<0.01$ ), which indicated that Akk was effective in improving the impairment of memory capacity in APP/PS1 mice.
In the open-field test, there were no significant differences when comparing the proportion of time spent in the central district among all groups (Fig. 4c). The clearing times of the APP/PS1-PBS mice were lower than those of the WT-PBS mice (Fig. $4 \mathrm{~d}, p<0.05$ ), but the clearing times were significantly increased in APP/PS1 mice treated with Akk than in the PBS treatment group. Only mice fed a normal diet showed a significant difference, which indicated that the gavage with Akk may have played a role in improving the ability of the mice to remain attentive. In addition, the duration of standing on the hind legs in the WT mice was higher than in the APP/PS1 mice when compared to the PBS intervention group, which did not show a significant difference after Akk treatment (Fig. 4e, $p<0.01$ ). In the APP/PS1 mice, Akk intervention significantly reduced the duration of standing in mice fed a normal diet, suggesting that Akk treatment could also help maintain curiosity regarding the surrounding environment, although the effect 
A
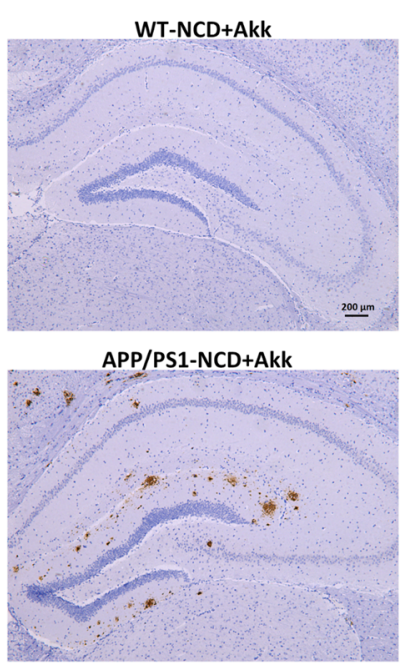

APP/PS1-HFD+Akk

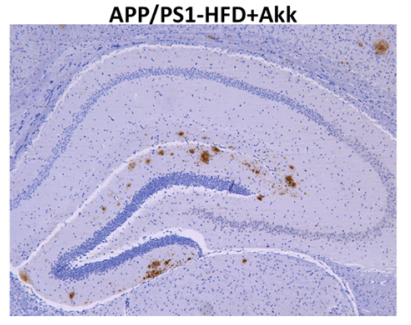

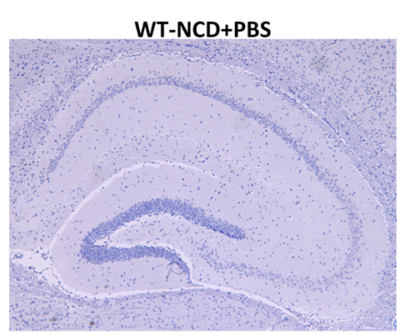

APP/PS1-NCD+PBS

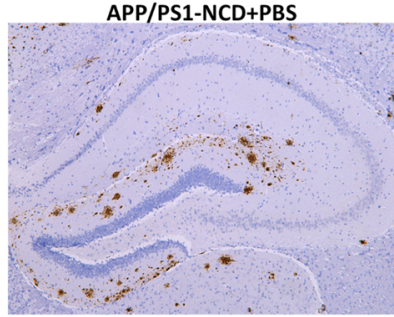

APP/PS1-HFD+PBS

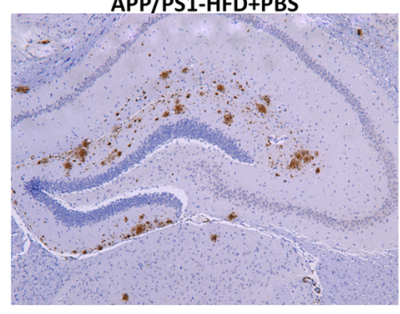

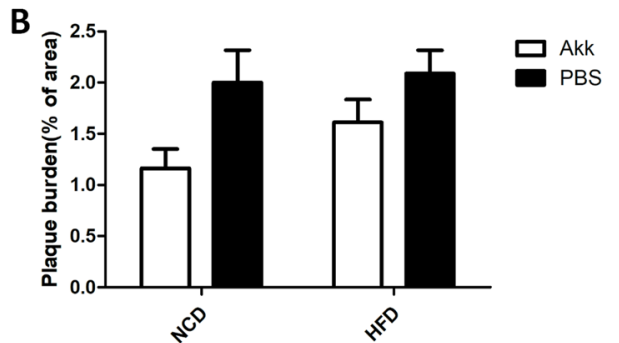

C

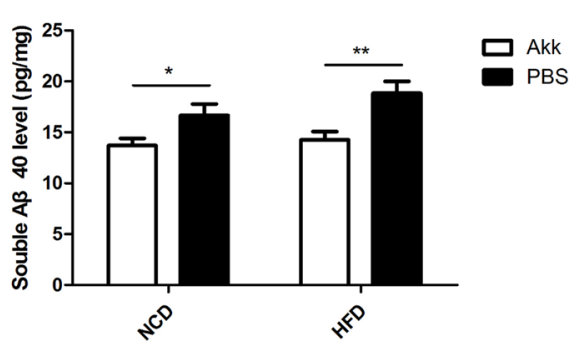

D

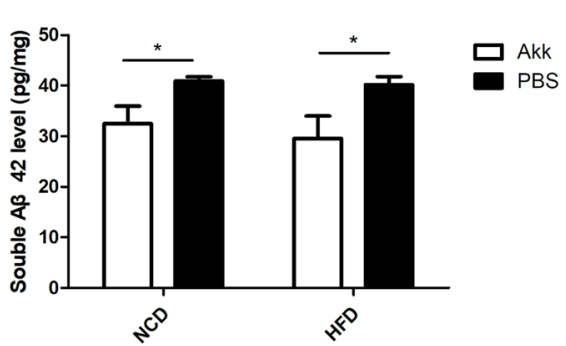

Fig. 3 Akkermansia muciniphila reduced $\mathbf{A} \beta$ plaque deposits and $\mathbf{A} \boldsymbol{\beta}$ levels in brains of APP/PS1 mice. a Representative $I H C$ images of the hippocampus from the mice. $\mathbf{b}$ Statistical analyses of the plaque burden. $\mathbf{c}$ Soluble A 40 and $\mathbf{d}$ A $\beta 42$ were analyzed by ELISA in the cortex of APP/PS1 mice. Data are presented as the mean \pm SEM, $N=6-10$ per group. ${ }^{*} p<0.05 ;{ }^{* *} p<0.01 ;{ }^{* * *} p<0.001$.

did not appear in the mice fed a high-fat diet. These data indicated that Akk could reduce the damage to spatial learning ability in APP/PS1 model mice.

\section{Discussion}

Recently, increasing numbers of studies have reported that a close relationship exists between gut microbiota and neurological disorders, which has been called the gutbrain axis ${ }^{29}$. Studies have shown that intestinal microbes have important implications in $\mathrm{AD}^{30}$. $\mathrm{AD}$ is a progressive degenerative disease of the central nervous system, mainly characterized as cognitive impairment, memory dysfunction, decreased self-care ability and decreased behavioral ability. There were major differences in the intestinal microflora composition between AD patients and normal subjects, suggesting that microbial composition may have a role in the development of brain $\mathrm{A} \beta$ amyloidosis ${ }^{24}$. The reduction in the abundance of Akkermansia was reported to be associated with obesity, type 2 diabetes, dysfunction of the intestinal barrier and other metabolic syndromes $^{31-33}$, most of which are important risk factors for AD. It has been further reported that the abundance of Akk in APP/PS1 mice decreased with age ${ }^{24}$. These reports suggest that supplementation with Akk could be a new strategy for the prevention and treatment of $\mathrm{AD}$. In the present study, with age, the Akk abundance decreased in APP/PS1 mice (Supplementary Fig. 1), and APP/PS1 mice showed a significant increase in fasting blood glucose levels and insulin resistance at 9 months of age, the mucus cells in the colon were significantly decreased and the serum DAO level was increased, indicating damaged intestinal barrier function. In addition, obvious impairment of cognitive function and learning memory ability, and an apparent accumulation of $A \beta$ protein in the brain were observed in 9-month-old APP/PS1 mice.

In the past decades, researchers believed that metabolic syndromes, including insulin resistance and obesity, were important risk factors for cognitive impairment and dementia $^{5}$. Studies had shown that insulin receptor expression was defective in the brains of $\mathrm{AD}$ patients and AD model animals. Studies also showed that IR substrate1 (IRS1) and IR substrate-2 (IRS2) expression were decreased, and the levels of inactivated serinephosphorylated IRS1 were increased, resulting in insulin resistance in the brain. Moreover, intestinal barrier destruction and endotoxemia were observed in AD model 


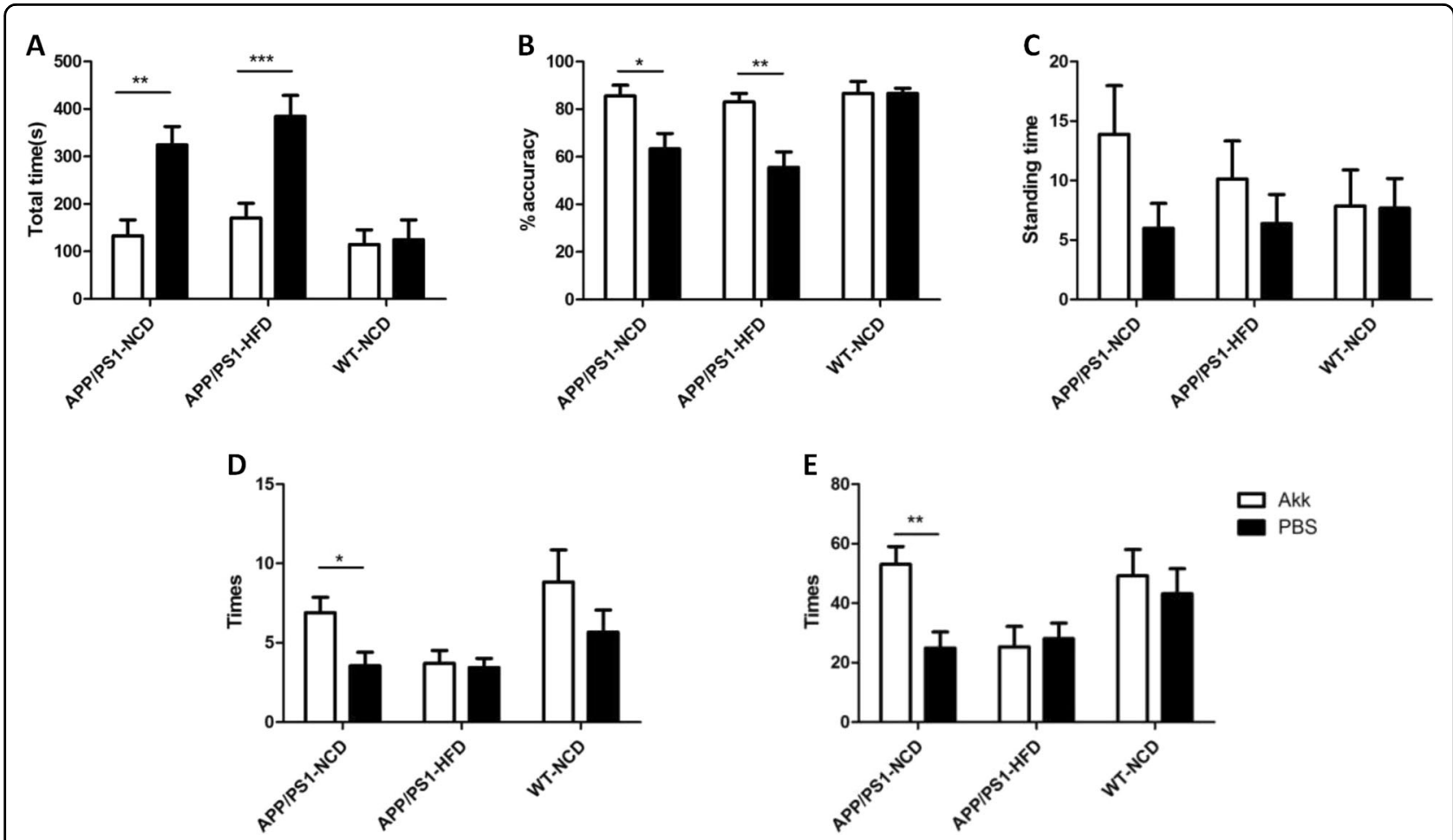

Fig. 4 Akkermansia muciniphila ameliorated cognitive impairment and anxiety-related behaviors in APP/PS1 model mice. a, $b$ The total time of learning and accuracy rate after $24 \mathrm{~h}$ of training in the $\mathrm{Y}$-maze tests. $\mathbf{c}-\mathbf{e}$ In the open-field tests, the time spent in the center and the total times of clearing and standing on the hind legs were monitored for each mouse. Data are presented as the mean $\pm \mathrm{SEM}, N=6-10$ per group. ${ }^{*} p<0.05 ;{ }^{* *} p<$ $0.01 ;{ }^{* * *} p<0.001$.

animals $^{20}$. Bacterial-derived LPS and amyloid protein can aggravate intestinal permeability, resulting in increased expression of cytokines and pro-inflammatory factors such as interleukin 17A (IL-17A) and IL-22, which can cross the intestinal barrier and blood brain barrier and cause an immune response in brain ${ }^{34}$. These studies indicated that $\mathrm{AD}$ may be a metabolic disease, and some scholars refer to it as type III diabetes ${ }^{5}$. Our study found that Akk could improve impaired fasting glucose and insulin resistance in $\mathrm{AD}$ model mice, increase the number of colonic mucus cells, decrease serum DAO levels and repair damaged intestinal barriers. Moreover, we found that compared to mice in the normal chow diet group, AD mice in the high-fat diet group have shorter colons, heavier weight, and higher cholesterol levels, liver steatosis is more severe (Supplementary Fig. 4). On the basis of our experimental results, Akk has a significant effect on alleviating the disease of AD mice, no matter in the high-fat diet group or the normal chow diet group, indicating that Akk has a protective role in $\mathrm{AD}$ disease under different conditions of abnormal lipid metabolism.

Epidemiological and clinical studies have shown that adults with obesity or diabetes have a much higher risk of developing dementia in the future. Obesity, insulin resistance and hyperlipidemia are forms of lipid toxicity, leading to inflammation, which may have a key role in neurological dysfunction. Cognitive impairment in the CNS could be attributed to changes in hippocampus structure and function in a portion of obesity-induced patients $^{35}$. Similarly, brain damage might also promote the development of obesity. We found that APP/PS1 mice showed significantly increased food intake, and the emergence of lipid metabolism disorders, such as increased levels of total cholesterol and triglycerides, liver steatosis, scapular brown adipocytes whitening, which were aggravated when treated with HFD. It is important to note that the above symptoms in APP/PS1 mice were improved after the mice were treated with Akk. Scapular brown adipocyte whitening could be seen in obese and ageing mice ${ }^{36-39}$, which was also observed in APP/PS1 mice in the course of our experiments. Our results demonstrated that Akk alleviated whitening of scapular brown adipocytes caused by a high-fat diet and aging. The reason may be that Akk can promote the utilization of free fatty acids and glucose in circulating blood, which can serve as a substrate to maintain the thermogenic activity of brown adipose tissue, so as to ensure the morphology and function of brown adipocytes ${ }^{37}$. Mice treated with PBS were unable to make full use of free fatty acids and 
glucose, resulting in severe accumulation of fat and elevated levels of blood glucose, brown adipocytes gradually replaced by white adipocytes, decreased thermogenesis and reduced energy consumption. These mice became obese and displayed liver steatosis. This hypothesis may also explain, to some extent, how Akk improved liver steatosis and metabolic syndrome. Consistent with this hypothesis, we found that Akk increased the expression of UCP1, which is a protein specifically expressed in brown adipose, which reflects the cellular thermogenic activity. The levels of UCP1were higher in mice treated with HFD. This phenomenon might be explained by diet-induced thermogenesis (DIT) ${ }^{40,41}$.We proffer that HFD stimulated thermogenesis in brown adipose tissue (BAT), and the mice displayed elevated BAT activity. Further study is needed to explore and verify this mechanism, however.

The main pathological features of $\mathrm{AD}$ are the formation of senile plaques (SP) and neurofibril tangles (NTFs) in the brain $^{42}$. Moreover, the main component of SP is $\beta$-amyloid (A $\beta)$, which has a crucial role in the occurrence and development of $\mathrm{AD}$, and is a common pathway for various factors to induce $A D^{43}$. $A \beta$ protein has an important role in the pathogenesis of AD. Self-clearing and degradation barriers of $A \beta$ protein lead to protein plaques. The $A \beta$ protein can reduce the clearance of $A \beta$ protein by macrophages, thereby producing an immune response $^{44}$. The $\mathrm{A} \beta$ protein can also promote neuronal apoptosis. There was a major difference in the composition of intestinal microflora between $\mathrm{AD}$ patients and normal subjects, suggesting that microbial composition may have a role in the development of brain $A \beta$ amyloi$\operatorname{dosis}^{24}$. It is worth mentioning that, in our study, $\mathrm{A} \beta$ protein deposition was reduced in the hippocampus and the cerebral cortex of APP/PS1 mice treated with Akk.

APP/PS1 mice also showed reduced attention to themselves and the environment after evaluation via the open-field test in our study, in agreement with previous studies $^{27}$, and these phenomena were alleviated by Akk treatment. The results of the Y-maze test showed that a high-fat diet further aggravated the impairment of learning and spatial memory ability in APP/PS1 mice. However, these symptoms were significantly improved with Akk treatment. These results suggest that Akk may increase mucus cells and promote the secretion of mucus to repair the impaired intestinal barrier, thereby reducing levels of LPS and other pro-inflammatory substances, improving metabolic levels, relieving insulin resistance in the brain, reducing the deposition of $A \beta$ protein, and protecting the nerves from damage.

In general, Akk treatment effectively improved intestinal barrier dysfunction, glucose and lipid metabolic disorders, and relieved cognitive impairment by reducing the level of $A \beta$ plaque deposition in the brain. These findings suggest that the ameliorative effect of Akk treatment on cognitive function can be attributed to the repair of metabolic disorders and, concomitantly, reduced incidence of $A \beta$ pathology. Our findings will provide a new insight that Akk supplementation is expected to be a new strategy for the prevention and treatment of $\mathrm{AD}$.

\section{Acknowledgements \\ The present study was supported by grants from the Guangdong Provincial Department of Science and Technology (2015A020213002), and the \\ Guangzhou Kangze Medical Science and Technology Co., Ltd Fund (201511).}

\section{Author details}

'Department of Laboratory Medicine, Zhu Jiang Hospital, Southern Medical University, 510282 Guangzhou, Guangdong, China. ${ }^{2}$ Jiangmen Central Hospital, Affiliated Jiangmen Hospital of Sun Yat-sen University, 529000 Jangmen, Guangdong, China. ${ }^{3}$ Transfusion Medicine, Zhu Jiang Hospital, Southern Medical University, 510282 Guangzhou, Guangdong, China

\section{Conflict of interest}

The authors declare that they have no conflict of interest.

\section{Ethical approval}

We confirm that manuscript has not been published elsewhere and is not under consideration by another journal for publication, in whole or in part. A single study has not been split into several parts to increase the quantity of to various journals or to one journal over time. None of the data or images have been fabricated or manipulated in order to support the conclusions. Consent to submit has been received explicitly from all co-authors, as well as from the responsible authorities. All authors have approved the manuscript and agree to submission of this manuscript for publication.

\section{Publisher's note}

Springer Nature remains neutral with regard to jurisdictional claims in published maps and institutional affiliations.

Supplementary Information accompanies this paper at (https://doi.org/ 10.1038/s41387-020-0115-8).

Received: 31 March 2019 Revised: 7 March 2020 Accepted: 18 March 2020 Published online: 22 April 2020

\section{References}

1. Kuhlmann, J., Andreasson, U. \& Pannee, J. CSF Aá1-42: an excellent but complicated Alzheimer's biomarker - a route to standardisation. Clin. Chim Acta 467, 27-33 (2016).

2. Stone, J. G. et al. Frontiers in Alzheimer's disease therapeutics. Ther. Adv. Chronic Dis. 2, 9-23 (2011).

3. Galimberti, D. \& Scarpini, E. Progress in Alzheimer's disease. J. Neurol 259 201-211 (2012).

4. Brookmeyer, R., Johnson, E., Ziegler-Graham, K. \& Arrighi, H. M. Forecasting the global burden of Alzheimer's disease. Alzheimers Dement. 3, 186-191 (2007).

5. Campos-Pena, V. et al. Metabolic syndrome as a risk factor for alzheimer's disease: is abeta a crucial factor in both pathologies? Antioxid. Redox Signal $\mathbf{2 6}$, 542-560 (2017)

6. Craft, S. Insulin resistance and Alzheimer's disease pathogenesis: potential mechanisms and implications for treatment. Curr. Alzheimer Res. 4, 147-152 (2007).

7. De Felice, F. G. Alzheimer's disease and insulin resistance: translating basic science into clinical applications. J. Clin Invest. 123, 531-539 (2013).

8. De Felice, F. G. Connecting type 2 diabetes to Alzheimer's disease. Expert Rev. Neurother. 13, 1297-1299 (2013). 
9. Craft, S. Insulin resistance syndrome and Alzheimer's disease: age- and obesityrelated effects on memory, amyloid, and inflammation. Neurobiol. Aging $\mathbf{2 6}$ 65-69 (2005).

10. Barbagallo, M. \& Dominguez, L. J. Type 2 diabetes mellitus and Alzheimer's disease. World J. Diabetes 5, 889-893 (2014).

11. Ho, L. et al. Diet-induced insulin resistance promotes amyloidosis in a transgenic mouse model of Alzheimer's disease. FASEB J. 18, 902-904 (2004).

12. Sims-Robinson, C., Kim, B., Rosko, A. \& Feldman, E. L. How does diabetes accelerate Alzheimer disease pathology? Nat. Rev. Neurol. 6, 551-559 (2010).

13. Carvalho, $C$. et al. Type 2 diabetic and Alzheimer's disease mice present similar behavioral, cognitive, and vascular anomalies. J. Alzheimers Dis. 35, 623-635 (2013).

14. Yaffe, K. et al. Metabolic syndrome and cognitive decline in elderly Latinos: findings from the Sacramento Area Latino Study of Aging study. J. Am. Geriatr. Soc. 55, 758-762 (2007)

15. Carvalho, C. et al. Metabolic alterations induced by sucrose intake and Alzheimer's disease promote similar brain mitochondrial abnormalities. Diabetes 61, 1234-1242 (2012).

16. De Felice, F. G. \& Lourenco, M. V. Brain metabolic stress and neuroinflammation at the basis of cognitive impairment in Alzheimer's disease. Front. Aging Neurosci. 7, 94 (2015).

17. Rios, J. A., Cisternas, P., Arrese, M., Barja, S. \& Inestrosa, N. C. Is Alzheimer's disease related to metabolic syndrome? A Wnt signaling conundrum. Prog. Neurobiol. 121, 125-146 (2014).

18. van Dijk, G. et al. Towards understanding brain-gut-microbiome connections in Alzheimer's disease. BMC Syst. Biol. 10, 63 (2016).

19. Moloney, A. M. et al. Defects in IGF-1 receptor, insulin receptor and IRS-1/2 in Alzheimer's disease indicate possible resistance to IGF-1 and insulin signalling. Neurobiol. Aging 31, 224-243 (2010).

20. Talbot, K. et al. Demonstrated brain insulin resistance in Alzheimer's disease patients is associated with IGF-1 resistance, IRS-1 dysregulation, and cognitive decline. J. Clin. Invest. 122, 1316-1338 (2012).

21. Ferreira, S. T., Clarke, J. R., Bomfim, T. R. \& De Felice, F. G. Inflammation, defective insulin signaling, and neuronal dysfunction in Alzheimer's disease. Alzheimers Dement. 10, S76-S83 (2014).

22. Rajilic-Stojanovic, M. \& de Vos, W. M. The first 1000 cultured species of the human gastrointestinal microbiota. FEMS Microbiol. Rev. 38, 996-1047 (2014).

23. Brahe, L. K. et al. Specific gut microbiota features and metabolic markers in postmenopausal women with obesity. Nutr. Diabetes 5, e159 (2015).

24. Harach, T. et al. Reduction of Abeta amyloid pathology in APPPS1 transgenic mice in the absence of gut microbiota. Sci. Rep. 7, 41802 (2017).

25. Everard, A. et al. Cross-talk between Akkermansia muciniphila and intestinal epithelium controls diet-induced obesity. Proc. Natl Acad. Sci. USA 110, 9066-9071 (2013).

26. Shin, N. R. et al. An increase in the Akkermansia spp. population induced by metformin treatment improves glucose homeostasis in diet-induced obese mice. Gut 63, 727-735 (2014).
27. Mao, Y. F. et al. Intranasal insulin alleviates cognitive deficits and amyloid pathology in young adult APPswe/PS1dE9 mice. Aging Cell 15, 893-902 (2016).

28. Guo, X. et al. Different subtype strains of Akkermansia muciniphila abundantly colonize in southern China. J. Appl. Microbiol. 120, 452-459 (2016).

29. Zhu, X. et al. Microbiota-gut-brain axis and the central nervous system. Oncotarget 8, 53829-53838 (2017).

30. Cattaneo, A. et al. Association of brain amyloidosis with pro-inflammatory gut bacterial taxa and peripheral inflammation markers in cognitively impaired elderly. Neurobiol. Aging 49, 60-68 (2017).

31. Dao, M. C. et al. Akkermansia muciniphila and improved metabolic health during a dietary intervention in obesity: relationship with gut microbiome richness and ecology. Gut 65, 426-436 (2016).

32. Grander, C. et al. Recovery of ethanol-induced Akkermansia muciniphila depletion ameliorates alcoholic liver disease. Gut 67, 891-901 (2018).

33. Plovier, H. et al. A purified membrane protein from Akkermansia muciniphila or the pasteurized bacterium improves metabolism in obese and diabetic mice. Nat. Med. 23, 107-113 (2017).

34. Pistollato, F. et al. Role of gut microbiota and nutrients in amyloid formation and pathogenesis of Alzheimer disease. Nutr. Rev. 74, 624-634 (2016).

35. O'Brien, P. D., Hinder, L. M., Callaghan, B. C. \& Feldman, E. L. Neurological consequences of obesity. Lancet Neurol. 16, 465-477 (2017).

36. Graja, A. \& Schulz, T. J. Mechanisms of aging-related impairment of brown adipocyte development and function. Gerontology 61, 211-217 (2015).

37. Bargut, T. C., Aguila, M. B. \& Mandarim-de-Lacerda, C. A. Brown adipose tissue: updates in cellular and molecular biology. Tissue Cell 48, 452-460 (2016).

38. Martins, F. F., Bargut, T., Aguila, M. B. \& Mandarim-de-Lacerda, C. A. Thermogenesis, fatty acid synthesis with oxidation, and inflammation in the brown adipose tissue of ob/ob (-/-) mice. Ann Anat. 210, 44-51 (2017).

39. Yin, F., Sancheti, H., Liu, Z. \& Cadenas, E. Mitochondrial function in ageing: coordination with signalling and transcriptional pathways. J. Physiol. 594, 2025-2042 (2016).

40. Liao, W. H., Henneberg, M. \& Langhans, W. Immunity-based evolutionary interpretation of diet-induced thermogenesis. CELL Metab. 23, 971-979 (2016).

41. Rothwell, N. J. \& Stock, M. J. A role for insulin in the diet-induced thermogenesis of cafeteria-fed rats. Metabolism 30, 673-678 (1981).

42. McGeer, P. L. \& McGeer, E. G. NSAIDs and Alzheimer disease: epidemiological, animal model and clinical studies. Neurobiol. Aging 28, 639-647 (2007).

43. Hill, J. M. \& Lukiw, W. J. Microbial-generated amyloids and Alzheimer's disease (AD). Front. Aging Neurosci. 7, 9 (2015).

44. Zhang, J., Ke, K. F., Liu, Z., Qiu, Y. H. \& Peng, Y. P. Th17 cell-mediated neuroinflammation is involved in neurodegeneration of abeta1-42-induced Alzheimer's disease model rats. PLOS ONE 8, e75786 (2013). 